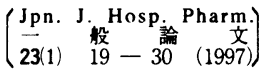

\title{
Characterization of Powdered Drugs by UV Absorption Spectrum in the Hospital Pharmacy
}

\author{
SHOJI INAGAKI* ${ }^{*}$, IKUO KOZAI $\dagger^{2}$, TATSUMORI TAKEDA $\dagger^{3}$ and MICHIO KOJIMA $\dagger^{1}$ \\ Department of Pharmacy, Mie University Hospital $\dagger^{1}$ \\ Department of Pharmacy, Hikone City Hospital $\dagger^{2}$ \\ Department of Pharmacy, Ueno City Hospital $\dagger^{3}$
}

\author{
$\left(\begin{array}{l}\text { Received June 17, } 1996 \\ \text { Accepted October 18, } 1996\end{array}\right)$
}

Characterization of powdered drugs depends on a visual inspection of the dispenser in most hospitals. And one of the most important problems of visual inspection is its lack of reliance that must be excluded from the actual preparation of the drug itself.

In this study, we attempted to develop a method to resolve this problem using a quick and easy procedure presenting greater reliability than the currently applied visual one.

Combining non-solvent sample preparation, opal glass absorption spectroscopy, baseline collection, the relative strengths of absorbance at three wavelengths ( $\mathrm{Qr}$ values), and a computer system, we developed a method by which characterization of drugs can be done quickly and easily, even if the subject mixture contains 2-3 kinds of drugs.

Characterization of drugs using the method has one principal defect, however, that it demands Qr values of pure components before use, unless the characteriation of drugs can not be accomplished from the Qr plot.

In spite of this fault, the Qr plot prepared by the method is useful for the characterization of drugs in a hospital pharmacy because even the calculation of the Qr plot is sufficient to demonstrate to the operator whether or not the preparation is correct.

Key words — quick characterization, powdered drug, opal glass spectroscopy, three wavelength spectroscopy, translucent sample, visual inspection

\section{Introduction}

It must be careful to treat powdered durgs in hospital pharmacy, because those drugs have few distinctive feature in appearance and it is more troublesome to characterize each other after their dispensation. Therefore, careless dispensation makes a serious problem frequently.

Usually, characterization of powdered drugs depends on a visual inspection of dispenser in most

$\dagger^{1}$ 三重県津市江戸橋 2 丁目174番地 ; 174, Edobashi 2-chome, Tsu-shi, Mie, 514 Japan

$\dagger^{2}$ 滋賀県彦根市本町 2-1-45; 2-1-45, Honmachi, Hikone, Shiga, 522 Japan

$\dagger^{3}$ 三重県上野市四十九町831; 831, Shijukumachi, Ueno, Mie, 518 Japan 
hospitals. This system has several merits such as quick and easy etc., so, it is introduced to most hospitals. But the visual characterization of a powdered drug demands a wide and reliable informations of drugs for the operator, and the one of the most important defect of the system is it lack of reliance that has to exclude from the preparation of drug.

In this study, we attempt to develop a method which procedure is quick and easy and its reliability is much more than the visual one.

In order to increase of the reliability with keeping the procedure quickly and easily, a opal glass ultraviolet absorption spectroscopy for powdered drug is applied in which solvent is excluded except a anti-dispertion reagent.

By using this spectroscopy, UV absorption spectra could be obtained directly from drugs or dispensed samples without other treatments such as dissolvation, separation etc.. But on the UV spectra obtained by the method, their baselines are represented in relative high absorbance region and peaks and valleies are shown as gently slopes, when it is compared with the conventional UV absorption spectra, although, their $\lambda_{\max }$ and $\lambda_{\min }$ exhibit normal values. Therefore, it is necessary to introduce other kinds of concept to characterize drugs from the dull spectra as well as the conventional one. For the purpose, three kinds of conception are introduced, that is, opal glass spectroscopy, baseline correction and three wavelengths absorption spectroscopy in which relative strength ( $\mathrm{Qr}$ ) of absorbances at three different wavelengths is applied. $\mathrm{Qr}$ is a parameter which is modified from a concept of Complementary Tristimulus Colorimetry, ${ }^{1-3)}$ and homologous to the trichromatic coordinates of the trichromatic system.

Combining non-solvent sample preparation, opal glass absorption spectroscopy ${ }^{4,5)}$, baseline collection, Qr parameter and computer system, we have developed a method by which characterization of drugs could be done quickly and easely by comparing Qr values of a sample and of the standard mixture, and even if the lack of the standard mixture (reference), it is possible to characterize $2 \sim 3$ kinds of drugs in mixture when a series of $\lambda_{\max }$ and it $\varepsilon$ of the drugs are known.

In order to verify the method, Sulpyrine (Sul.), Phenacetin (Phe.), Caffeine (Caf.) and their mixtures are used as a fundamental exsample.

\section{Theoretical Discussion}

\section{Opal Glass Absorption Spectroscopy}

In conventional spectrophotometry, samples for absorption spectrum measurement must be transparent solution. But samples which are prepared by this method, become to translucent by reason of nonsolvent system, even if, anti-dispertion reagent is applied.

Generally, when a translucent sample is analyzed by the conventional spectrophotometry, a large part of the projected light is scattered in the sample, then the amount of light that enter into phototube is small. As a result of the phenomenon, ratio of sample to reference comes to small value, and it causes a less characteristic spectrum. Therefore, an opal glass is usally used to improve the ratio, that is, it decreases the amount of transmitted light from reference cell which is transparent, on the contrary, amount of scattered light from sample cell which is translucent, is less effected by the opal glass and 
as a result, the more charasteristic absorption spectrum is obtained from a translucent sample.

\section{Correction of Baseline}

In spectra of translucent samples, baseline lies in high absorbance region on the shorter wavelength range and gradually decline toward the zero line according to scan to longer wavelength.

This phenomenon is proper to a translucent sample and it due to the lack of uniformity in emission energy of light source, so the decline of baseline occurs in proportion to the energy of light source and to degree of translucence.

The energy of light source at every wavelength in spectrophotometer consider to be constant, therefore, the baseline could be estimated from its reference spectra by appling the quintic curve fitting method.

And the correction could be done by shifting down baseline to zero line parallel to vertical axis, at which wavelength components exhibit no light absorption.

\section{Three Wavelength Opal Glass Absorption Spectroscopy (TOAS Method)}

In absorption spectroscopy, characterization of component depends on its $\lambda_{\max }, \lambda_{\min }, \varepsilon$ of spectra when it is pure, but in the case of complex system such as mixture, translucent sample etc., it is difficult to identify them each other by those parameters. Three wavelength opal absorption spectroscopy in which absorbance is measured by opal glass method, is applied for the complex system to chracterize drugs. This method is reduced from Complementary Tristimulus Colorimetry, and is simplified to adapt the method for hospital pharmacy use.

A set of absorbances are measured at three definite wavelengths (generally called $u, v$ and w) which are specific wavelength for the drug. (Fig. 1)

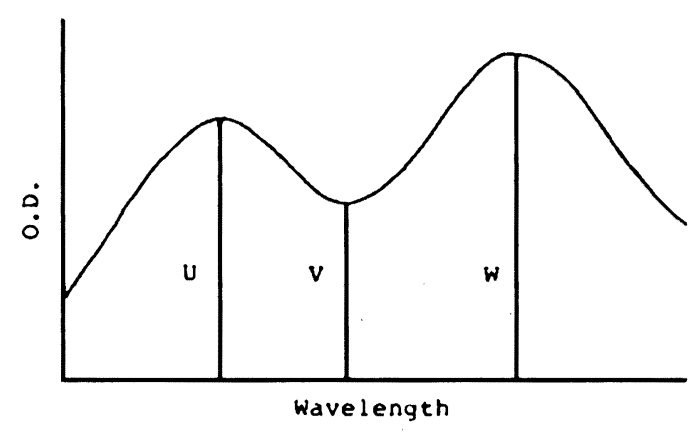

Fig. 1. Selection of Three Wavelengths

O. D. : Optical Density usually is' expressed as Absorbance Value of Spectrophotometer

The ratio of the absorbance at each wavelength to the sum ( $u, v$ and $w$ ) of the each absorbance is calculated and named them $\mathrm{Qr}$ point $(\mathrm{Qr})$.

$$
\begin{aligned}
& \mathrm{Qr}=\mathrm{Rr} / \Sigma \cdot \mathrm{Rr}=\mathrm{Rr} / \mathrm{J} \\
& \mathrm{J}=\Sigma \cdot \mathrm{Rr} \cdots \ldots \ldots \ldots \ldots \ldots \ldots \ldots \ldots \ldots \ldots \ldots \ldots \ldots \ldots \ldots \ldots \ldots
\end{aligned}
$$

Here, $\mathrm{Rr}$ : absorbance at the $\mathrm{r}$ wavelength, Qr : Qr value at the $r$ wavelength, $J:$ sum of the absorbances at three wavelengths, E: overall absorptivity, C: molarity, $1:$ length of the light path, $r:$ any of the three wavelengths ( $u, v$ and $w$ ).

As the Qr value is a relative strength of a absorbance to sum of three absorbances, its value is independent for its concentration and its length of light path. If the same set of wavelength for $u, v$ and $w$ are used, same drug shows same $Q r$ value so, consequently, $Q r$ is a characteristic parameter for the drug. And a drug could be shown as a single point on an orthogonalcoordinates in which two kinds of $\mathrm{Qr}$ value from three ( $\mathrm{u}, \mathrm{v}$ and $\mathrm{w})$ are nominated. And then, the graph of $\mathrm{Qu}-\mathrm{Qv}(\mathrm{Qu}-\mathrm{Qw}$ or 
$\mathrm{Qv}-\mathrm{Qw}$ ) is called Qr plot.

Qr point of the mixture that contains a drug and b drug, is represented as a point on the straight line which terminals are $\mathrm{Qr}$ points of each drugs ( $\mathrm{a}$ and $\mathrm{b}$ ) respectively ${ }^{1-3)}$. And molar fraction of a drug (qa) and $b$ drug (qb) can be quantified as the following equations (4) ( 6 ).

$$
\begin{aligned}
& \mathrm{qa}=\mathrm{Ca} /(\mathrm{Ca}+\mathrm{Cb}) \\
& \mathrm{qb}=\mathrm{Cb} /(\mathrm{Ca}+\mathrm{Cb})=1-\mathrm{qa} \\
& \mathrm{qa}=\mathrm{Eb}(\mathrm{Qrb}-\mathrm{Qrm}) /\{\mathrm{Ea}(\mathrm{Qrm}-\mathrm{Qra})+\mathrm{Eb}(\mathrm{Qrb}-\mathrm{Qrm})\}
\end{aligned}
$$

Qr point of the mixture that contains three kinds of drug ( $a, b$ and $c$ ) is presented as a point in triangle which a pexes are $\mathrm{Qr}$ points of each drugs ( $\mathrm{a}, \mathrm{b}$ and $\mathrm{c}$ ). And ratios of $\mathrm{Ca}$ to $\mathrm{Cb}, \mathrm{Ca}$ to $\mathrm{Cc}$ and $\mathrm{Ca}$ to $\mathrm{Cb}$ to $\mathrm{Cc}$ are represented as the following equations $(7) \sim(9)$ respectively.

Here, $\mathrm{Ca}, \mathrm{Cb}$ and $\mathrm{Cc}$ are the concentrations of drug $\mathrm{a}, \mathrm{b}$ and $\mathrm{c}$ and Qrm is a Qr point of the mixture.

$$
\begin{aligned}
& \mathrm{Ca}: \mathrm{Cb}=\mathrm{qa}: 1-\mathrm{qa} \\
& \mathrm{Ca}: \mathrm{Cc}=\mathrm{qa}^{\prime}: 1-\mathrm{qa} \text { ' } \\
& \mathrm{Ca}: \mathrm{Cb}: \mathrm{Cc}=\mathrm{qa}^{\prime} \cdot \mathrm{qa}^{\prime}:(1-\mathrm{qa}) \cdot \mathrm{qa}^{\prime}:\left(1-\mathrm{qa}^{\prime}\right) \cdot \mathrm{qa}
\end{aligned}
$$

Therefore, the weight of drug $\mathrm{a}, \mathrm{b}$ and $\mathrm{c}$ can be calculated from the above equations, if the total weight of the sample or a concentration of one of the three drugs. (Fig. 2)

( Two Drug System)

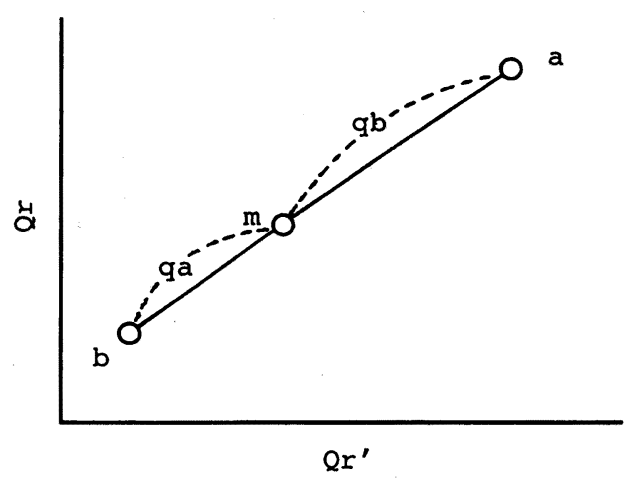

( Three Drug System)

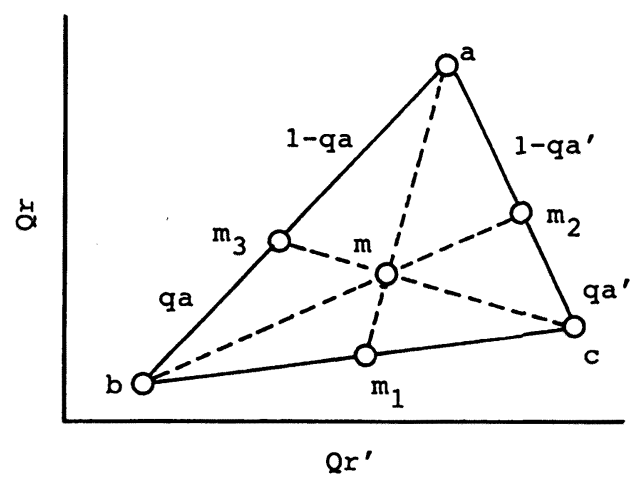

Fig. 2. Qr Plot of Two and Three Drug Systems and Determination of Molar Concentration from the Mixture $m$ by the Graphical Method

\section{Experimental}

\section{Materials}

The drugs used were Sulpyrine (Daiichi Seiyaku Co., Ltd., official preparation, Lot No.BL 442), Phenacetin (Nakakita Yakuhin Co., Ltd., official preparation, Lot No.130287), Caffeine (Astra Japan Co., Ltd., official preparation, Lot No.1350), Aminopyrine (Nakakita Yakuhin Co., Ltd., Lot No.190874), Chlorpheniramin Mal. (Sankyo Co., Ltd., official preparation, Lot No. M035L), Acetazolamide (Lederle 
(Japan), Ltd., official preparation, Lot No.519-2), Primidon (Dainippon Pharmaceutical Co., Ltd., official preparation, Lot No.5912) and Nitrazepam (Shionogi Seiyaku Co., Ltd., official preparation, Lot No. A350431).

Purification was not done before use. $\mathrm{KBr}$ tablets ${ }^{6}$ were produced using a special grade reagent of potassium bromide.

\section{Equipment}

Absorption spectra were measured using Shimadzu MPS-2000. The cell for TOAS method is used a pair of quarz plates $(3 \mathrm{~cm} \times 3 \mathrm{~cm})$. The cell holders are attached to the surface of photomultiplier.

A system for TOAS method is presented in Fig. 3. By the system, it is done quickly with accuracy to correct the baseline, to calculate Qr points and the concentation of the components, to display the results on CRT tube and to print it out.

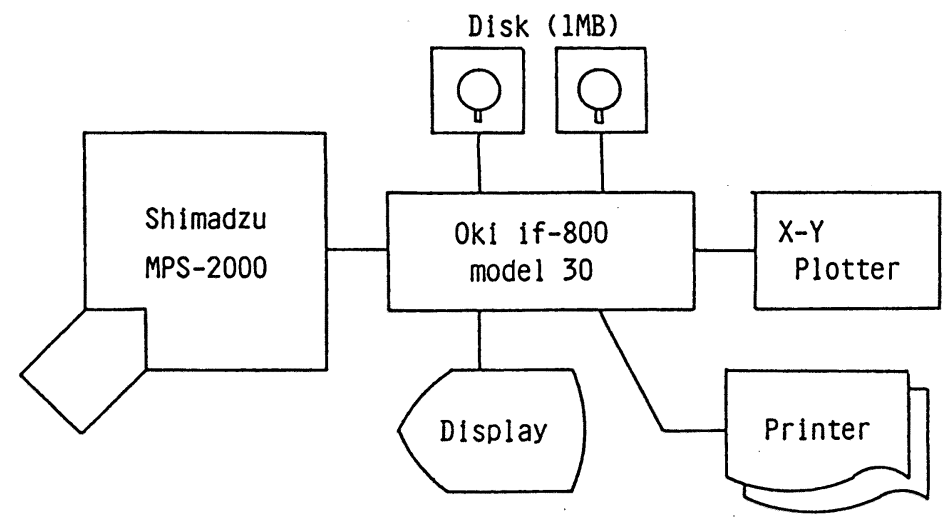

Fig. 3. Block Diagram of Measurement System for Three Wavelength Opal Glass Absorption Spectroscopy (TOAS Method)

\section{Operation Procedure}

1 ) Prepatation of Sample

An adepuate volume (ca. $0.1 \mathrm{mg}$ ) of sample and anti-dispersion reagent (Nujol, ca. $100 \mu \mathrm{l}$ ) are put on the quartz plate and rubbed each other by another quarz plate. After rubbing well, the pair of plate adheres each other and set it on the cell holder of the opal glass spectrophotometer. Reference is a $\mathrm{KBr}$ pellet which is used in IR absorption spectrum. To adjust the concentration of sample, sample or anti-dispersion reagent is added proportionally to the degree of absorbance. The result of TOAS is outputed by the printer.

2) Absorption Spectra

Spectra of Sul., Phe., Caf. and their mixture were measured in translucent state by TOAS. (Fig. 4 )

3 ) Selection of Wavelength

In Sul.- Phe.- Caf. system optical density are measured at $220 \mathrm{~nm}$ for $\mathrm{u}$ range, $250 \mathrm{~nm}$ for $\mathrm{v}$ range, $310 \mathrm{~nm}$ for $\mathrm{w}$ range and $350 \mathrm{~nm}$ for baseline correction respectively. And in Ami-- Phe.- Chl. system and Ace.- Pri.- Nit. system, optical density are measured at $218 \mathrm{~nm}$ for u range, $250 \mathrm{~nm}$ for 

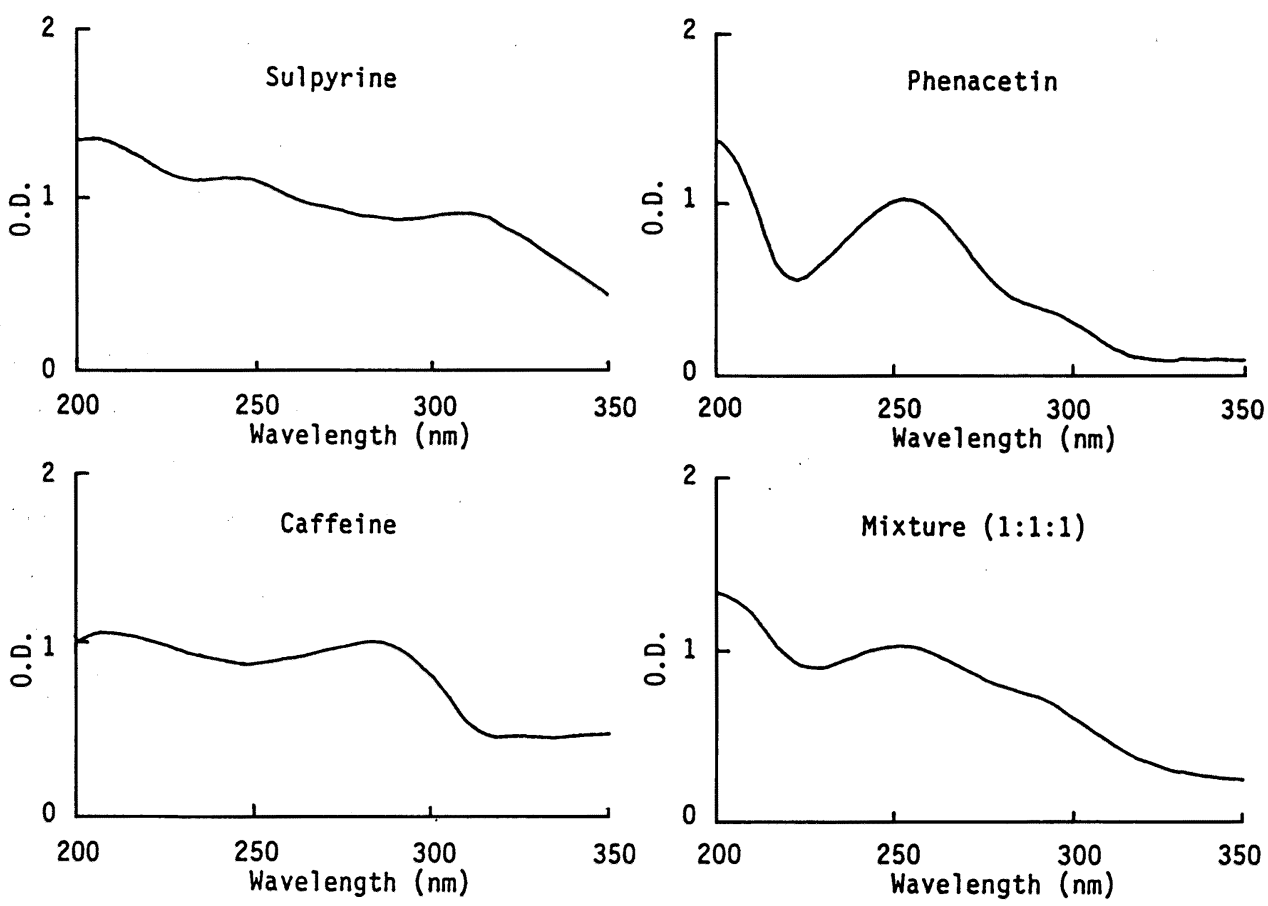

Fig. 4. Absorption Spectra of Sulpyrine, Phenacetin, Caffeine and Their Mixture in Translucent State by TOAS Method

O. D. : Optical Density

O. D. is usually expressed as Absorbance Value of Spectrophotometer

$\mathrm{v}$ range and $280 \mathrm{~nm}$ for $\mathrm{w}$ range, and $226 \mathrm{~nm}$, for $\mathrm{u}$ range, $257 \mathrm{~nm}$ for $\mathrm{v}$ range and $310 \mathrm{~nm}$ for $\mathrm{w}$ range respectively.

4) Correction of Baseline

In spectra which are measured by opal glass absorption spectrophotometer, the baseline lies a relative high absorbance region, so, it must be shifted down to zero at a wavelength at which any component of sample exhibits no light absorption, in parallel to vertical axis.

After the shift down, whole baseline is estimated by a quintic curve fitting method using microcomputer.

\section{Results and Discussion}

\section{Quick Procedure in Hospital Pharmacy}

In hospital, it is very important factor to shorten a working time without reduction its accuracy. We thought that visual inspection with accuracy is the better time saving way. To carry out the principle, a spectrometoric inspection is developed instead of visual one.

There are some problems to convert the visual way into the conventional spectrometry, that is, the former depends on a relative strength of the three primary colors, so is selective and specific but is dull in evaluation of concentration, on the contrary, the latter is insufficient for expression of the sense 
of sight, although, evaluation of the concentration is accurate. So, TOAS which bases on relative strength of absorbance, is usefull for inspection of drugs. And by a aid of microcomputer system, the inspection procedure in hospital will be more quickly with accuracy.

\section{Spectrometry without Solvents}

It is usefull for shortening a working time to cut off some pretreatment such as dissolition, extraction, filtration etc.. In this study, nonsolvent spectrometry is introduced, as a result of it, the procedure is simplified but spectrum must by measured under a translucent state. By application of TOAS for translucent sample, spectra could be measured even if in nonsolvent system. (Fig. 4 )

\section{Correction of Baseline}

The absorpion spectra of Lactose which is basic substance of preparation, and of Lactose with antidispersion reagent are measured at various degree of turbidity. And as a reference, $\mathrm{KBr}$ tablet is also measured. (Fig. 5 )

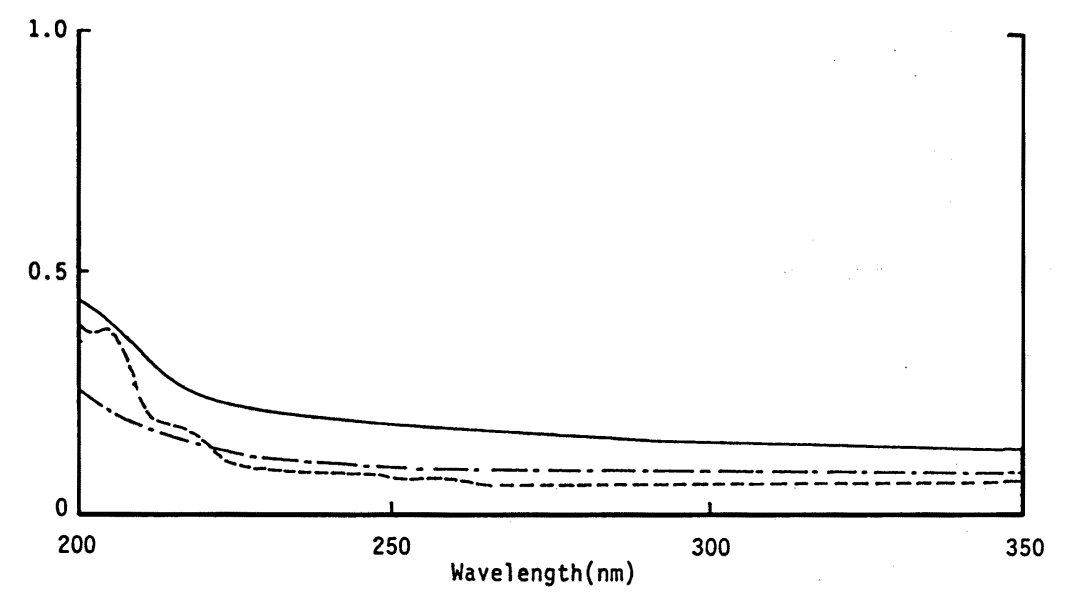

Fig. 5. Baseline of Translucent Sample Using the Quartz Plate Method, Nujol Method and $\mathrm{KBr}$ Method [- : Lactose ;--- : $\mathrm{KBr} ;{ }^{----}:$Nujol and Lactose]

Those spectra have a similar tendency in shape although, the back ground absorption (baseline) are different except in a wavelength range shorter than $215 \mathrm{~nm}$. Therefore, it is possible to estimate baseline of a basic preparation substances by the procedure above mentioned in longer than $215 \mathrm{~nm}$ and to measure absorption spectra from spectra obtained by the opal glass absorption spectrophotometer by subtracting absorbance of baseline from absorbance of sample.

In this method, it is impossible to estimate the absorption spectra when its basic preparation substances exhibit any absorption band in UV region or large amount of anti-dispertion reagent is used.

\section{Qr plot of the Mixture}

Utilizing TOAS method, Qr values of Sul., Phe. and Caf. are obtained from translucent state using equations $(1)-(3)$.

And comparison of $\mathrm{Qr}$ values in transparent state with in traslucent state is shown in Table 1. 
Table 1. Qr Values of Sulpyrine, Phenacetin, and Caffeine in Translucent and in Transparent State by the Three Wevelength Opal Glass Absorption Spectroscopy

(TOAS Method)

\begin{tabular}{ccccccc}
\hline Name & \multicolumn{3}{c}{ Translucent s. } & & \multicolumn{3}{c}{ Transparent s. } \\
& Sul. & Phe. & Caf. & Sul. & Phe. & Caf. \\
\hline $\begin{array}{c}\text { Qr value } \\
\text { range }\end{array}$ & 0.370 & 0.401 & 0.504 & & & \\
$(220 \mathrm{~nm})$ & 0.366 & 0.405 & 0.498 & 0.385 & 0.388 & 0.512 \\
\hline $\mathrm{V}$ & 0.372 & 0.395 & 0.508 & & & \\
$\begin{array}{c}\text { range } \\
(250 \mathrm{~nm})\end{array}$ & 0.343 & 0.553 & 0.396 & & & \\
& 0.338 & 0.560 & 0.399 & 0.314 & 0.567 & 0.389 \\
$\mathrm{~W}$ & 0.288 & 0.549 & 0.393 & & & \\
range & 0.296 & 0.035 & 0.103 & 0.301 & 0.045 & 0.099 \\
$(310 \mathrm{~nm})$ & 0.283 & 0.056 & 0.099 & & & \\
\hline
\end{tabular}

Table 2. Comparison of Qr Values of Standard Samples and Preparation Sample in Transparent State by the Three Wevelength Opal Glass Absorption Spectroscopy (TOAS Method)

(Aminopyrine, Phenacetin, Chlorpheniramin Mal. and their Mixture)

\begin{tabular}{|c|c|c|c|c|}
\hline Name & Conc. $\left(\times 10^{-5} \mathrm{~mol}\right)$ & $\begin{array}{c}Q u \\
(218 \mathrm{~nm})\end{array}$ & $\begin{array}{l}\text { Qr } \\
Q v \\
(250 \mathrm{~nm})\end{array}$ & $\begin{array}{c}Q W \\
(280 \mathrm{~nm})\end{array}$ \\
\hline Aminopyrine & 5.38 & 0.34 & 0.338 & 0.322 \\
\hline Phenacetin & 3.79 & 0.143 & 0.749 & 0.108 \\
\hline Chlorpheniramin Mal. & 7.33 & 0.808 & 0.166 & 0.026 \\
\hline Standard Mix. $\quad\left[\begin{array}{l}\text { Ami. } \\
\text { Phe. } \\
\text { Chl. }\end{array}\right.$ & $\begin{array}{l}1.8 \\
4.8 \\
4.1\end{array}$ & 0.207 & 0.621 & 0.172 \\
\hline Preparation Mix. $\left[\begin{array}{l}\text { Ami. } \\
\text { Phe. } \\
\text { Chl. }\end{array}\right.$ & $\begin{array}{l}1.8 \\
4.8 \\
4.1\end{array}$ & 0.209 & 0.618 & 0.173 \\
\hline
\end{tabular}


Table 3. Comparison of Qr Values of Standard Samples and Preparation Sample in Transparent State by the Three Wevelength Opal Glass Absorption Spectroscopy (TOAS Method)

(Acetazolamide, Primidone, Nitrazepam and their Mixture)

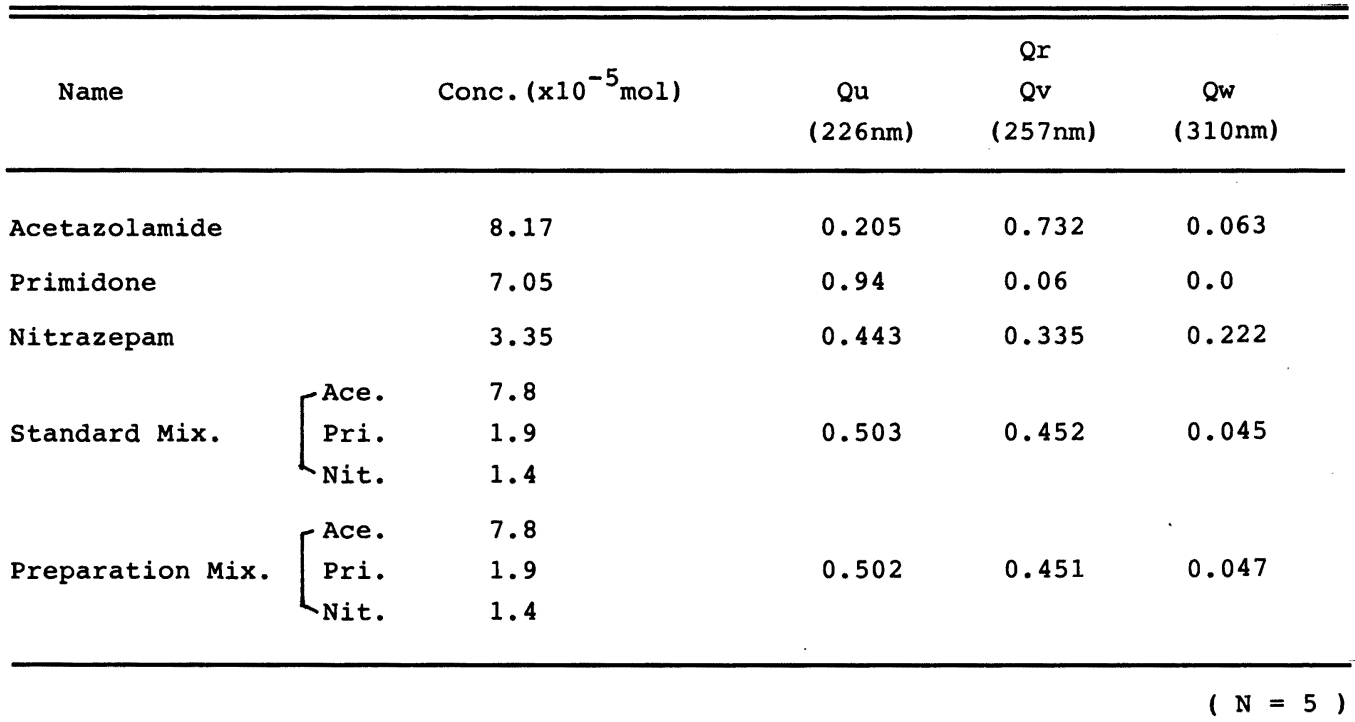

Similarly, the comparison of Qr values of Ami.- Phe.- Chl. system and Ace.- Pri.- Nit. system in translucent state are shown in Table 2 and Table 3 respectively.

From the data, the following results are noticed, that is, Qr can be obtained from a translucent sample, although those values deviate each other slightly $( \pm 2 \%)$. But the deviation is regarded as negligible for the characterization of a drug. There is some difference of $\mathrm{Qr}$ values between from transparent state and from translucent, and it is cosidered that the difference is caused mainly by the correction of baseline in translucent state.

\section{Characterization from the Qr Plot}

As a prescription of a standard sample, Sul.1.0 g, Phe.1.0 g and Caf. $0.2 \mathrm{~g}$ is used and Qu-Qv plot is drawn up in Fig. 6.

In the Qr plot (Fig.6), Point ( 1 ) is the Qr of the standard preparation (Sul.1.0g, Phe.1.0g, and Caf. $0.2 \mathrm{~g}$ ). And point Sulpyrine, Phenacetin and Caffeine are the Qr point for Sul., Phe. and Caf. respectively. It is indicated that Qr plot of the preparation mixture is similar to the standard mixture. And the preparation mixture is correct.

\section{Information from the Qr Plot}

Mixtures of two kinds of drug among Sul., Phe. and Caf. are represented as points $(3)-(5)$ in Qr plot (Fig.6). Point (3), (4) and (5) are the Qr point in the cases of ratio of Sul., to Phe., Phe. to Caf. and Caf. to Sul are 1:1 respectively. Those points $(3)-(5)$ are on the line which terminals are Qr points of each drugs. And if Qr point of a mixture is on the line, it indicates that the mixture is composed of two kinds of drug which are represented as the terminal points of line in 


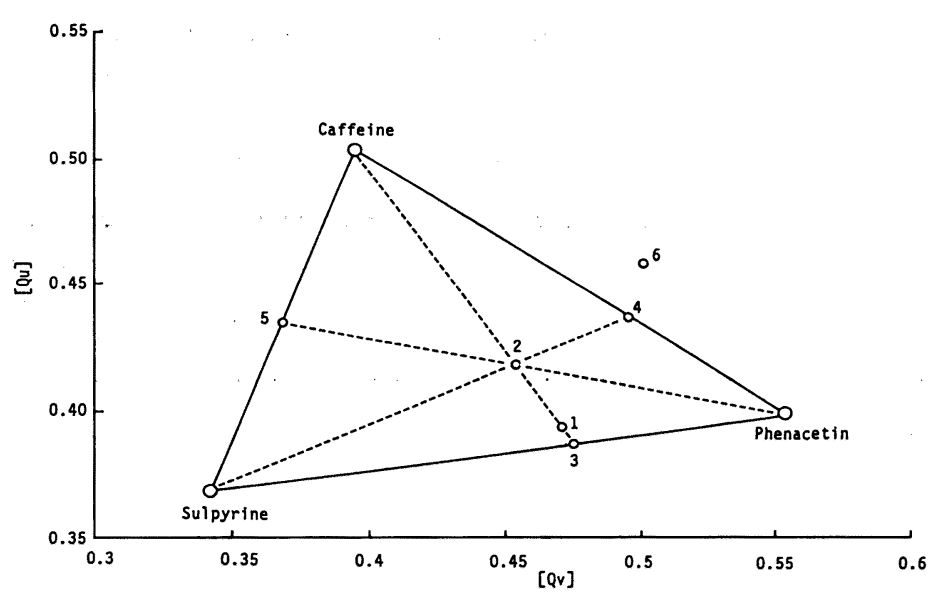

Fig. 6. Qu-Qv Plot of Sulpyrine, Phenacetin, Caffeine and Their Mixtures by the Three Wavelength Opal Glass Absorption Spectroscopy (TOAS Method) Composition ratio---- Sul. : Phe. : Caff. : Asp.

$\begin{array}{rllllllll}\text { NO. } 1 & 1 & : & 1 & : & 0.2 & & \\ 2 & 1 & : & 1 & : & 1 & & \\ 3 & 1 & : & 1 & & & & \\ 4 & & & 1 & : & 1 & & \\ 5 & 1 & & : & & 1 & & \\ 6 & & & 1 & : & 0.2 & : & 1\end{array}$

Qr plot. If a operator dose not dispense one drug among the standard prescription, the Qr point lies on the circumference of triangle. And it can be identified from the Qr plot which drug did not dispensed.

In the case of three components system, the characterization of drugs could be done by utilizing Qr plot and its values. As a example, characterization of one drug that is incorrect in weighting during its preparation, although other procedures are correct. Point (2) represents Qr point of the misdispensed preparation at which dose of Caf. is wrong in weighting. This preparation containes $1.0 \mathrm{~g}$ of $\mathrm{Caf}$. in stead of its correct weight is $0.2 \mathrm{~g}$. The disagreement of Qr point (1) and (2) indicates that the preparation [2] differs from standard [1] in composition.

In Qr plot, three Qr points (sandard preparation, incorrect preparation and one pure among three) are on line, if one drug is misdispensed.

In Fig. 6, point $(1),(2)$ and Caffeine are on line (Caffeine-point 3), it indicates that $(1)$ and (2) are consist of Caf. and preparation [3] which is consisted of Sul. and Phe.. So, (1) and (2) are regarded as mixtures of Caf. and (3). And it is estimated that preparation [2] differ with [1] in content of $\mathrm{Caf}$.

\section{Estimation of the Weight of Drugs}

By using Qr Values, the ratio of Caf. in (1) and (2) could be estimated from equations (4) $-(8)$, and it is known that how many weight of drug did misdispensed. 
Table 4. Determination of Mixture (Sulpyrine, Phenacetin, and Caffeine) Using the Three Wavelength Opal Glass Absorption Spectroscopy(TOAS Method)

\begin{tabular}{|c|c|c|c|c|c|c|c|c|}
\hline \multirow{2}{*}{ No. of Qr plot } & \multicolumn{5}{|c|}{ Component Ratio } & \multicolumn{3}{|c|}{ Molar Ratio (q) } \\
\hline & sul. & $:$ & Phe. & $:$ & Caf. & sul. & Phe. & Caf. \\
\hline 1 & 1 & : & 1 & : & 0.2 & 0.314 & 0.584 & 0.102 \\
\hline 2 & 1 & : & 1 & : & 1 & 0.222 & 0.438 & 0.34 \\
\hline 3 & 1 & : & 1 & & & 0.319 & 0.691 & \\
\hline 5 & 1 & & : & & 1 & 0.34 & & 0.66 \\
\hline
\end{tabular}

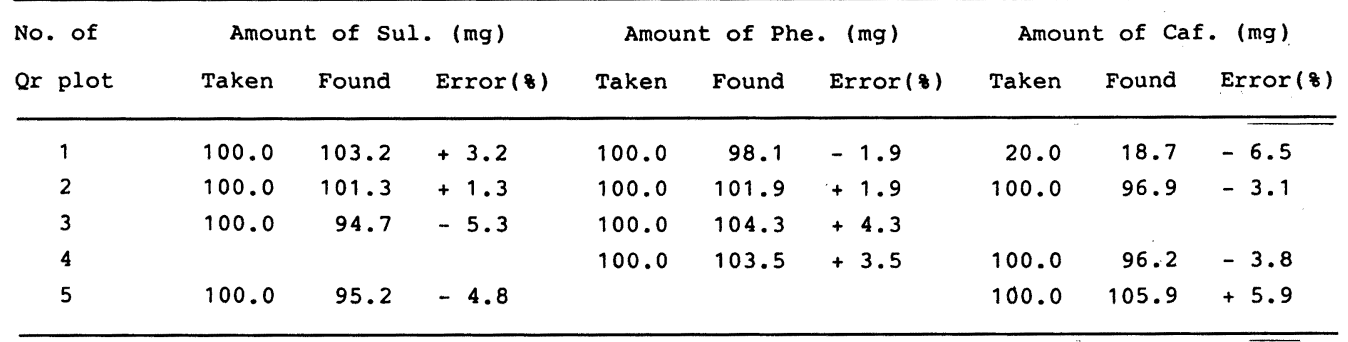

[ No. of Qr plot correspond to No. of Qv-Qu plot.]

[ $\mathrm{N}=5$ ]

It is difficult to characterize a preparation in which two or three kinds of drug are misdispensed, by the Qr plot alone. In that case, characterization of each drug could be done by a determination of their components by utilizing the Qr values and equations $(4)-(8)$. The results of the determination are shown Table 4. It is a little troublesome and deviated in its calculated values (about 10\%), although this method is adaptable for every case.

It is detectable from Qr plot that other drug is dispensed instead of correct one. For example, (6) point shows the Qr point when Sul. is replaced by Aspirin. Qr point of the mixture which contains another kind of drug instead of the correct one, is represented as apoint outside the triangle produced by connecting the Qr points for the three drugs.

Charaterization of drugs by the TOAS method has some defects, that is, it demands Qr values of pure components before use, unless the characterization of drugs can not be done from the Qr plot, and it is unreliable to deal with a preparation which contains over five kind of drugs by the Qr plot, because the $\mathrm{Qr}$ values from a translucent sample deviate from each other. So, in some complex mixture, Qr points can not able to see any difference clearly from its Qr plot. But in spite of the fault, Qr plot prepared by TOAS method is usefull for the characterization of drugs in hospital pharmacy, because, even a glance over the Qr plot it is enough to show the operator that the preparation is correct or not.

In this study, a standard preparation for TOAS method is examined in UV region. For next study, TOAS method will be developed to utilize absorption spectra in visible and IR region, and to increasing its detectable limit of small amount of drug in preparation. 


\section{References}

1) H. Flaschka, Talanta, 7,90-106(1960).

2) C. N. Reilly and H. Flaschka, Anal. Chem., 32,1218-1232(1960) ; C. N. Reilly and E. M. Smith, ibid., 32,1233-1240(1960).

3 ) S. Hirose, I. Ikeuchi and K. Sakakibara, Bunseki Kagaku, 21,183-189(1972); S. Yoshida, K, Oda and S. Hirose, Chem. Pharm. Bull., 32, 1011-1017(1984).

4 ) K. Shibata, "Supekutoru Sokutei To Bunkokodokei, "Kodan-Sha, Tokyo, 1974, p. 156-165.

5 ) F. Yoshino and H.Osawa, "Rinsho-Seikagaku-Kensa Ni Okeru Bunko-Sokuteiho," Gakkai Press Center, 1984 , p. 19-20.

6 ) R. M. Silverstein, G. C. Bassler and T. C. Morrill, "Spectrometric Identification of Organic Compounds," 3 rd ., John Wiley \& Sons, New York, 1974, p. 75-78. 